\title{
Parâmetros reprodutivos após indução de estro com diferentes intervalos de permanência do dispositivo vaginal em cabras Toggenburg acíclicas
}

\author{
[Reproductive parameters after induction of estrus with different ranges of the vaginal
} device in acyclic Toggenburg goats]

\author{
P.M.P. Nascimento-Penido ${ }^{1}$, F.Z. Brandão ${ }^{1}$, A.O. Penido ${ }^{2}$, P.F.V.Pereira ${ }^{1}$, J.F. Fonseca ${ }^{3}$ \\ ${ }^{1}$ Faculdade de Veterinária - Universidade Federal Fluminense - Niterói, RJ \\ ${ }^{2}$ Empresa de Pesquisa Agropecuária de Minas Gerais - Belo Horizonte, MG \\ ${ }^{3}$ Embrapa Caprinos e Ovinos - Coronel Pacheco, MG
}

\begin{abstract}
RESUMO
Este estudo objetivou avaliar o comportamento sexual e a dinâmica ovulatória de cabras da raça Toggenburg após a indução do estro com dispositivo intravaginal de progesterona durante seis, nove e 12 dias de permanência. No momento da inserção do dispositivo e 24 horas antes da retirada do dispositivo, foi administrado $5 \mathrm{mg}$ de dinoprost e 200UI de eCG, respectivamente. A dinâmica ovulatória foi acompanhada por ultrassonografia a cada oito horas, enquanto o estro foi observado a cada 12 horas. Todas as cabras apresentaram estro. O intervalo da retirada do dispositivo ao início do estro foi de 29,5 \pm 9,6 para $\mathrm{G}_{6 \text { dias }} ; 34,0 \pm 6,0$ e $32,4 \pm 7,7 \mathrm{~h}, \mathrm{G}_{9 \text { dias }}$ e $\mathrm{G}_{12 \text { dias, }}$, respectivamente. Igualmente, foi encontrada diferença $(\mathrm{P}<0,05)$ entre o $\mathrm{G}_{6 \text { dias }}$ e os outros grupos. A duração do estro diferiu $(\mathrm{P}<0,05)$ entre o $\mathrm{G}_{6 \text { dias }}$ $(36,0 \pm 12,6)$ e os outros dois grupos $\left(\mathrm{G}_{9 \text { dias }}: 31,2 \pm 14,3 ; \mathrm{G}_{12 \text { dias }}: 33,4 \pm 8,6\right)$. A taxa de ovulação $(81 \%$; $89,5 \%$ e; 71,4\%), intervalo da retirada do dispositivo à ovulação $(50,5 \pm 11,4 ; 46,3 \pm 5,9$ e; $46,7 \pm 8,3)$, diâmetro do maior folículo $(6,6 \pm 0,3 ; 6,6 \pm 0,9$ e; $6,9 \pm 0,8)$ diâmetro do segundo maior folículo $(6,4 \pm$ $0,7 ; 6,6 \pm 0,8$ e; $6,8 \pm 0,6)$ e o número de folículos ovulados $(1,5 \pm 0,6 ; 1,7 \pm 0,6$ e 2,0 $\pm 0,9)$ para $\mathrm{G}_{6 \text { dias, }}$, $\mathrm{G}_{9 \text { dias }}$ e $\mathrm{G}_{12 \mathrm{dias}}$, respectivamente, foram semelhantes ( $\left.\mathrm{P}>0,05\right)$. Todos os protocolos foram eficientes em induzir o estro sincronizado em cabras da raça Toggenburg, trabalhando em conjunto com a eficiência reprodutiva e produtiva desses animais.
\end{abstract}

Palavras-chave: indução de estro, ciclicidade, cabras leiteiras, toggenburg, tratamento hormonal

\begin{abstract}
This study aimed to evaluate the sexual behavior and the ovulatory dynamics of Toggenburg goats after induction of synchronized estrus by an intravaginal progesterone device for six, nine and twelve days. At the device insertion and $24 \mathrm{~h}$ before the device removal, 5mg of dinoprost and 200 UI of eCG was administered, respectively. The ovulatory dynamics was assessed by ultrasound every $8 h$, while the sexual behavior was observed every $12 \mathrm{~h}$. All goats showed estrus. The intervals from device removal to estrus were $29.5 \pm 9.7,34.0 \pm 6.0$ and $32.4 \pm 7.7 \mathrm{~h}$ to $G_{6 \text { days }}, G_{9 d a y s}$ and $G_{12 d a y s}$, respectively. Iqually, it was found difference $(P<0.05)$ among $G_{6 d a y s}$ and the other groups. The duration of estrus differed $(P<0.05)$ among the $G_{6 d a y s}(36.0 \pm 12.6)$ and the other two groups $\left(G_{9 \text { days }}: 31.2 \pm 14.3 ; G_{12 d a y s}: 33.4 \pm 8.6\right)$. The ovulation rate (81.0\%; $89.5 \%$ and; $71.4 \%)$; the interval from device removal to ovulation (50.5 \pm 11.4 ; $46.3 \pm 5.9$ and; $46.7 \pm 8.3)$, the diameter of the largest follicle $(6.6 \pm 0.3 ; 6.6 \pm 0.9$ and; $6.9 \pm 0.8)$, diameter of the second largest follicle (6.4 $\pm 0.7 ; 6.6 \pm 0.8$ and; $6.8 \pm 0.6)$ and the number of ovulations $(1.5 \pm 0.6 ; 1.7 \pm 0.6$ and; $2.0 \pm 0.9)$ for $G_{6 d a y s}, G_{9 d a y s}$ and $G_{12 \text { days }}$, respectively, were similar $(P>0.05)$. All treatments were effective for the induction of synchronized estrus in Toggenburg goats, working jointly with the reproductive and productive efficiency of these animals.
\end{abstract}

Keywords: estrus induction, ciclicity, dairy goats, toggenburg, hormonal treatment

Recebido em 7 de abril de 2017

Aceito em 10 de julho de 2017

E-mail: paulampn@gmail.com 


\section{INTRODUÇÃO}

Nos últimos anos, muitos estudos vêm sendo realizados com protocolos hormonais para a indução de estro sincronizado em cabras Toggenburg, uma vez que elas vêm se destacando no mercado leiteiro por sua produção, fácil adaptabilidade e docilidade. Os tratamentos hormonais permitem induzir o estro sincronizado na fêmea em anestro e sincronizar o estro na fêmea cíclica, concentrando partos para períodos específicos, assim como a produção leiteira distribuída ao longo do ano, evitando, desse modo, perdas econômicas e déficit no leite (Zambrini, 2006; Amorim et al., 2008; Pietroski et al., 2013).

A influência do fotoperíodo é marcante tanto nos machos quanto nas fêmeas de raças oriundas do hemisfério norte, que iniciam seu ciclo reprodutivo anual em função da diminuição da intensidade de luz diária, marcadamente no outono. Esses animais são considerados animais poliéstricos estacionais de "dias curtos" ou "fotoperíodo negativo" (Traldi et al., 2007).

Para se driblar esse fotoperíodo, diferentes tratamentos à base de substâncias hormonais exógenas, seja para controlar a fase lútea, seja para induzir ou aumentar a resposta ovariana, estão sendo testadas. Esses tratamentos facilitam a utilização de uma alimentação racional e a observação dos partos, diminuindo a mortalidade perinatal. Permitem também a concentração de partos programados, gerando leite em todos os períodos do ano e lotes de cabritos para o abate mais homogêneo no momento da venda (Zambrini, 2006).

Dessa forma, o objetivo deste estudo foi avaliar o efeito da duração do tratamento hormonal (seis, nove e 12 dias) com implante intravaginal contendo progesterona, em fêmeas da raça Toggenburg, no período de anestro estacional, sobre a dinâmica ovulatória, bem como o comportamento sexual. Ademais, avaliou-se a influência das categorias animais (nulíparas, pluríparas lactantes e pluríparas secas) e o tipo de acasalamento sobre as respostas aos protocolos de indução do estro.

\section{MATERIAL E MÉTODOS}

O projeto foi aprovado junto ao Comitê de Ética em Pesquisa Animal da Universidade Federal Fluminense, sob o protocolo de número 0043808 .

O estudo foi realizado durante a estação de anestro estacional (novembro e dezembro), em uma propriedade comercial produtora de leite caprino, localizada na região da Zona da Mata mineira, Brasil (latitude $21^{\circ} 35^{\prime} \mathrm{S}$, longitude $43^{\circ}$ $15^{\prime} \mathrm{W}$ e altitude de $\left.484 \mathrm{~m}\right)$. O clima foi caracterizado como mesotermal Cwa, de acordo com a classificação Koppen, com inverno seco e verão quente.

Os animais foram mantidos em sistema de confinamento em baias coletivas, sob fotoperíodo natural e temperatura ambiente, alimentados no cocho com capim napier picado (Pennisetum purpureum v. Taiwan), concentrado, formulado e misturado na própria granja, água e sal mineral (Salminas Caprinos ${ }^{\circledR}$ Nutriplan - Brasil) ad libitum.

Foram selecionadas 62 cabras da raça Toggenburg, sendo estas de distintas categoriais animais: nulíparas $(n=20)$, pluríparas lactantes $(\mathrm{n}=21)$ e pluríparas secas $(\mathrm{n}=21)$, após a realização dos exames clínicos geral e reprodutivo específico e ultrassonográfico, seguidos da avaliação do escore da condição corporal (1 a 5 - Suiter, 1994).

Os animais foram divididos uniformemente quanto as categorias em três grupos experimentais de acordo com o tempo de permanência do implante intravaginal impregnado com progesterona: $6\left(\mathrm{G}_{6 \text { dias }}-\mathrm{n}=22\right)$, $9\left(\mathrm{G}_{9 \text { dias }} \mathrm{n}=19\right)$ ou $12\left(\mathrm{G}_{12 \text { dias }} \mathrm{n}=21\right)$. Todos os animais receberam no Dia 0 injeção paravulvar de $5 \mathrm{mg}$ de dinoprost (Lutalyse ${ }^{\circledR}$ - Zoetis, Brasil) e 24 horas antes da retirada do dispositivo foi administrado (i.m.) 200UI de eCG (Novormon ${ }^{\circledR}$ Zoetis, Brasil).

O estro foi observado duas vezes ao dia, pela manhã e à noite, (sete e 19 horas), com auxílio de rufiões. As fêmeas foram consideradas em estro quando apresentaram edema de vulva, agitação à presença do macho, como bater de cauda, micção frequente, procura pelo macho e aceitação da monta. 
O acompanhamento da dinâmica ovulatória iniciou-se no momento da retirada do implante intravaginal por meio de avaliações ultrassonográficas Modo-B, sendo repetidas a cada oito horas até a identificação das ovulações. Utilizou-se aparelho portátil (Aloka SSD 500, Aloka Co. Japão), equipado com transdutor linear de $5 \mathrm{MHz}$, por via transretal. Os ovários foram mapeados, e todos os folículos antrais com diâmetro $\geq 3 \mathrm{~mm}$ visibilizados foram mensurados. $\mathrm{O}$ diâmetro médio (média do comprimento e largura) de cada folículo foi calculado, sendo repetido a cada oito horas. A ovulação foi considerada como sendo o momento em que $\mathrm{o}(\mathrm{s})$ folículo(s) dominante(s) (FD), anteriormente identificado(s), não $\operatorname{estava(m)~}$ mais presente(s) na imagem ultrassonográfica, ou dependendo da irregularidade da parede do FD.

Para realização das análises estatísticas, utilizouse o programa SAEG 8.1 (Sistema para Análises Estatísticas e Genéticas), desenvolvido pela Universidade Federal de Viçosa, e o programa InStat 3.

Para as variáveis paramétricas (intervalo entre a retirada do dispositivo à IA; duração do estro; intervalo da retirada do dispositivo ao início do estro; intervalo da retirada do dispositivo à ocorrência da ovulação; intervalo do início do estro à ocorrência da ovulação; diâmetro do maior e do segundo maior folículo; número de folículos ovulados/animal) entre as categorias estudadas, aplicou-se a análise de variância, e para a comparação de mais de duas médias, utilizou-se o teste de Student-Newman-Keuls (SNK).

As variáveis não paramétricas: percentual de cabras em estro e taxa de animais ovulando foram submetidas ao teste de qui-quadrado.

\section{RESULTADOS}

Todos os animais manifestaram estro $(100 \%$ $62 / 62$ ), independentemente da categoria; $80,6 \%$ (50/62) das cabras demonstraram estro pela manhã e nenhuma antes de 24 h, após a retirada do implante.

A duração do estro apresentou diferença $(\mathrm{P}<0,05)$ entre a categoria das fêmeas: as nulíparas $(27,4 \pm 8,2 \mathrm{~h})$ demonstraram estro mais precocemente do que as pluríparas lactantes $(32,7 \pm 8,0 \mathrm{~h})$ e pluríparas secas $(33,4 \pm 7,5 \mathrm{~h})$.

A Tab. 1 apresenta os resultados referentes aos parâmetros do comportamento sexual estudados.

Os parâmetros ultrassonográficos estão apresentados na Tab. 2. Obteve-se diferença $(\mathrm{P}>0,05)$ apenas quanto ao intervalo do início do estro à ocorrência da ovulação, em que o tipo de acasalamento, monta natural (51,7 \pm 7,6 horas), demonstrou maior intervalo do que a IA $(48,0 \pm$ $9,8)$.

Tabela1. Parâmetros do comportamento sexual de cabras Toggenburg submetidas a protocolos de indução e sincronização de estro em estação de anestro estacional (média \pm desvio-padrão) com CIDR ${ }^{\circledR}$ durante seis, nove e 12 dias

\begin{tabular}{|c|c|c|c|c|}
\hline \multirow{2}{*}{ Variáveis } & \multicolumn{4}{|c|}{ Tratamentos experimentais } \\
\hline & 6 dias & 9 dias & 12 dias & Total \\
\hline Animais em estro (\%) & $\begin{array}{c}100 \% \\
(22 / 22)\end{array}$ & $\begin{array}{c}100 \% \\
(19 / 19)\end{array}$ & $\begin{array}{c}100 \% \\
(21 / 21)\end{array}$ & $\begin{array}{c}100 \% \\
(62 / 62)\end{array}$ \\
\hline $\begin{array}{l}\text { Intervalo entre a retirada do } \\
\text { dispositivo e o início do estro } \\
\text { (horas) }\end{array}$ & $29,5 \pm 9,7^{b}$ & $34,0 \pm 6,0^{\mathrm{a}}$ & $32,4 \pm 7,7^{\mathrm{a}}$ & $31,9 \pm 8,0$ \\
\hline Duração do estro (horas) & $36,0 \pm 12,6^{\mathrm{a}}$ & $31,2 \pm 14,3^{\mathrm{b}}$ & $33,4 \pm 8,6^{\mathrm{b}}$ & $33,6 \pm 11,9$ \\
\hline
\end{tabular}

Letras diferentes na mesma linha diferem $(\mathrm{P}<0,05)$. 
Tabela 2 Parâmetros ultrassonográficos avaliados durante a dinâmica ovulatória de cabras Toggenburg submetidas a protocolos de indução e sincronização de estro em estação de anestro estacional (média \pm desvio-padrão) com CIDR ${ }^{\circledR}$ durante seis, nove e 12 dias

\begin{tabular}{|c|c|c|c|c|}
\hline \multirow{2}{*}{ Variáveis } & \multicolumn{4}{|c|}{ Tratamentos experimentais } \\
\hline & 6 dias & 9 dias & 12 dias & Total \\
\hline Taxa de animais ovulando (\%) & $81 \%(18 / 22)^{a}$ & $89,5 \%(17 / 19)^{\mathrm{a}}$ & $71,4 \%(15 / 21)^{a}$ & $80,6 \%(50 / 62)$ \\
\hline $\begin{array}{l}\text { Intervalo da retirada do dispositivo } \\
\text { à ovulação (horas) }\end{array}$ & $50,5 \pm 11,4^{\mathrm{a}}$ & $46,3 \pm 5,9^{\mathrm{a}}$ & $46,7 \pm 8,3^{\mathrm{a}}$ & $47,8 \pm 8.9$ \\
\hline $\begin{array}{l}\text { Intervalo do início do estro à } \\
\text { ovulação (horas) }\end{array}$ & $29,8 \pm 12,7^{\mathrm{a}}$ & $24,4 \pm 6,2^{\mathrm{a}}$ & $22,9 \pm 7,1^{\mathrm{a}}$ & $25,7 \pm 9,4$ \\
\hline $\begin{array}{l}\text { Número de folículos } \\
\text { ovulados/animal }\end{array}$ & $1,5 \pm 0,6^{\mathrm{a}}$ & $1,7 \pm 0,6^{\mathrm{a}}$ & $2,0 \pm 0,9^{\mathrm{a}}$ & $1,7 \pm 0,7$ \\
\hline
\end{tabular}

Letras diferentes na mesma coluna diferem $(\mathrm{P}<0,05)$.

\section{DISCUSSÃO}

Todos os animais entraram em estro, demonstrando que os tratamentos foram eficientes na indução. Segundo Knight (2001), o período mínimo de exposição a $\mathrm{P}_{4}$ necessário para induzir o comportamento de estro varia de três a cinco dias. Assim, o período de utilização mínimo de $\mathrm{P}_{4}$, no estudo, foi eficiente para induzir o comportamento estral.

Fonseca et al. (2005), após tratarem animais não lactantes com esponjas impregnadas com $60 \mathrm{mg}$ de MAP por seis e nove dias, descreveram $89,5 \%$ e $84,2 \%$, respectivamente. Outros autores obtiveram taxas entre $91,3 \%$ e $98 \%$ após a utilização de 60mg de MAP (Baril et al., 1996; Menchaca e Rubianes, 2002).

Os resultados demonstraram $80,6 \%$ (50/62) dos animais com comportamento estral pela manhã. Relatos semelhantes em animais de mesma raça podem ser observados na literatura (Fonseca et al., 2005; Souza et al., 2011; Pietroski et al., 2013). Esse evento pode estar relacionado à secreção concentrada em ritmo noturno da melatonina, devido às horas de escuridão, resultando em um número maior de animais demonstrando estro pela manhã (Nagy et al., 2000; Fonseca e Simplício, 2008).

O período compreendido entre a retirada do dispositivo e o início do estro demonstrou intervalo mais curto no tratamento seis dias $(29,5$ $\pm 9,7 \mathrm{~h})(\mathrm{P}<0,05)$ do que os tratamentos nove dias $(34,0 \pm 6,0 \mathrm{~h})$ e 12 dias $(32,4 \pm 7,7 \mathrm{~h})$ (Tab. 1). Isso porque o tratamento seis dias se caracteriza por uma alta concentração de progesterona no final do tratamento, o que leva a uma brusca queda das concentrações desse esteroide após a retirada do dispositivo. Dessa forma, permite descarga mais intensa de FSH e LH, induzindo a maturação folicular, o aumento nos índices de $17 \_\beta$-estradiol e a ocorrência precoce do estro (Rubianes et al., 1998). Souza et al. (2011) relataram período semelhante $(33,1 \pm 1,9 \mathrm{~h})$, reutilizando $\mathrm{CIDR}^{\circledR}$ durante seis e 12 dias em animais da mesma raça. Por outro lado, resultados inferiores $(25,7 \mathrm{~h})$ foram descritos em animais da raça Saanen, durante o anestro estacional, utilizando MAP durante seis, nove e 12 dias (Pietroski et al., 2013), demonstrando que pode haver efeito de raça sobre o período da retirada do dispositivo e início do estro. O intervalo da retirada do implante ao início do estro está relacionado com o momento da administração do eCG. Muitos são os fatores que podem interferir neste intervalo da retirada do implante ao início do estro, como temperatura do ambiente, fase do ciclo estral no momento da indução do estro, tipo de acasalamento, nutrição, diferentes fontes de progestágenos, uso de gonadotrofinas e presença de reprodutores após a remoção do dispositivo intravaginal e dominância social (Baril et al., 1996; Orihuela, 2000; Dogan et al., 2008).

A duração do estro diferiu $(\mathrm{P}<0,05)$ de acordo com o tratamento $(36,0 \pm 12,6 \mathrm{~h} ; 31,2 \pm 14,3 \mathrm{~h}$; $33,4 \pm 8,6 \mathrm{~h}$ - seis, nove e 12 dias, respectivamente), assim como em relação à categoria. Cabras nulíparas $(27,4 \pm 8,2 \mathrm{~h})$ demonstraram duração do estro mais curta $(\mathrm{P}>0,05)$ do que as plulíparas lactantes $(32,7 \pm$ $8,0 \mathrm{~h})$ e secas $(33,4 \pm 7,5 \mathrm{~h})$. Isso pode ter ocorrido devido à presença de anticorpos antieCG produzidos pelas pluríparas, uma vez que estas já haviam sido submetidas a outro protocolo de indução e sincronização de estro. Maffili et al. (2006) obtiveram resultados semelhantes na estação de acasalamento natural 
com cabras Toggenburg tratadas durante cinco dias com dois dispositivos intravaginais diferentes, $\mathrm{CIDR}^{\circledR}(37,0 \pm 4,51 \mathrm{~h})$ e esponjas impregnadas com $60 \mathrm{mg}$ de MAP $(35,0 \pm 5,89 \mathrm{~h})$. Já em cabras Saanen tratadas com esponjas impregnadas com 60mg de MAP, durante seis $(33,7 \pm 13,6$ horas $)$ e nove dias $(29,3 \pm 10,6$ horas) (Fonseca et al., 2005) ou seis, nove e 12 dias (24,9h) (Pietroski et al., 2013), nas estações de acasalamento e anestro estacional, observouse diferença. Esse fato também pode estar relacionado a diversas razões, como a raça, fatores ambientais e nutrição.

No presente estudo, 80,6\% (50/62) dos animais ovularam. O mesmo valor foi relatado em cabras da raça Alpina (80\%, Fonseca et al., 2010) e Saanen (Pietroski et al., 2013) durante anestro estacional, demonstrando que independentemente do período de permanência do dispositivo intravaginal impregnado com progesterona, estes são eficientes para a indução da ovulação durante o anestro estacional.

O número de ovulações por animal foi de 1,7 \pm 0,7 , não demonstrando diferença entre o período de exposição ao progestágeno e a categoria dos animais $(\mathrm{P}>0,05)$. Os resultados assemelham-se a outros estudos do grupo com cabras Toggenburg reutilizando $\operatorname{CIDR}^{\circledR}(1,7 \pm 0,1$ ovulações, Souza et al., 2011), ou com a utilização de 60mg MAP em cabras Saanen utilizando MAP (1,6 \pm 0,2 ovulações, Pietroski et al., 2013), cabras da raça Alpina (1,7 \pm 0,1 ovulações, Fonseca et al., 2010).

Os diâmetros do maior folículo ovulado $(6,7 \pm$ $0,1 \mathrm{~mm})$, assim como o do segundo maior $(6,6 \pm$ $0,2 \mathrm{~mm})$, não diferiram $(\mathrm{P}>0,05)$ entre os tratamentos e a categoria, o que está de acordo com prévios trabalhos, que relataram um ou dois folículos em cada onda folicular maiores que $5 \mathrm{~mm}$ de diâmetro em cabras (Ginther e Kot, 1994).

A literatura demonstra diferença entre os trabalhos referentes ao diâmetro dos folículos pré-ovulatórios: 7,0mm (De Castro et al., 1999); $7,8 \mathrm{~mm}$ em cabras Murciano-Granadina (Gonzalez-Bulnez et al., 2004); 5,5mm em cabras Anglo Nubiana (Tenório Filho et al., 2007); 7,4mm em cabras Toggenburg (Souza et al., 2013) e 5,7mm em cabras Saanen (Pietroski et al., 2013). Porém, Nascimento (2009) relata que, durante a estação de acasalamento natural, os animais da mesma raça submetidos às mesmas variáveis (tratamentos e categoria) demonstraram diâmetro inferior $(6,1 \pm 0,6$ e 5,9 $\pm 0,5$; maior e segundo maior folículo) ao descrito no presente estudo. Logo, pode-se inferir que a raça e a estação estão diretamente relacionadas com o diâmetro em que o folículo ovula.

\section{CONCLUSÃO}

Tratamentos hormonais utilizando dispositivos impregnados com progestágeno, nos períodos de seis, nove e 12 dias, são eficientes para a indução e a sincronização de estro, assim como na ovulação durante o anestro estacional de cabras leiteiras. Dessa forma, colaboram para melhorar a eficiência reprodutiva de caprinos leiteiros, independentemente da categoria animal.

\section{AGRADECIMENTOS}

À Dra. Marlene Bruschi e ao Dr. Henrique Bruschi (in memorian); ao CNPq e à Embrapa Gado Leite, pelo suporte durante o estudo. Felipe Zandonadi Brandão e Jeferson Ferreira da Fonseca são bolsistas do CNPq.

\section{REFERÊNCIAS}

AMORIM, E.A.M.; TORRES, C.A.A.; FONSECA, J.F. et al. Dinâmica follicular em cabras da raça Toggenburg em lactação tratadas ou não com somatotropina bovina recombinante. Arq. Bras. Med. Vet. Zootec., v.59, p.1500-1508, 2008.

BARIL, G.; REMY, B.; LEBOEUF, B. et al. Syncronization of estrus in goats: the relationship between eCG binding in plasma, time of occurrence of estrus and fertility following artificial insemination. Theriogenology, v.45, p.1553-1559, 1996.

DE CASTRO, T.; RUBIANES, E.; MENCHACA, A. et al. Ovarian dynamics sérum estradiol and progesterone concentrations during the interovulatory inerval in goats. Theriogenology, v.52, p.399-411, 1999.

DOGAN, I.; KONYALI, A.; GUNAY, U. et al. Comparision of the effect of cronolone sponges and PMSG or cloprostenol on estrous induction in Turkish Saanen goats. Pol. J. Vet. Sci., v.11, p.29-24, 2008 . 
FONSECA, J.F.; BRUSCHI, J.H.; SANTOS, I.C.C. et al. Induction of estrus in non-lactanting dairy goats with different estrous synchrony protocols. Anim. Reprod. Sci., v.85, p.117-124, 2005.

FONSECA, J.F.; SIMPLÍCIO, A.A. Inseminação artificial e transferência de embriões em caprinos e ovinos. ENCONTRO INTERNACIONAL DA PECUÁRIA DA AMAZÔNIA, 1., 2008, Belém. Anais... Belém: AMAZONPEC, 2008.

FONSECA, J.F.; SOUZA, J.M.G.; BRUSCHI, J.H. et al. Induction of estrus in cyclic Alpine goats with short-term progestagen protocols with or without eCG administration. Rep. Fertil. Dev., v.22, p169, 2010. (Abstract).

GINTHER, O.J.; KOT, K. Follicular dinamics during the ocularoty season in goats. Theriogenology, v.42, p.987-1001, 1994.

GONZALEZ-BULNES, A.; MORENO, J.S.; GOMEZ-BRUNET, A. et al. Follicular dynamics during the oestrous cycle in dairy goats. Anim. Sci., v.68, p.547-554, 2004.

KNIGHT, T.W. Ram induced stimulation of ovarian and oestrous activity in anoestrus ewes a review. Proc. N. Z. Soc. Anim. Prod., v.43, p.711, 2001.

MAFFILI, V.V.; TORRES, C.A.A.; BRUSCHI, J.H. et al. Indução de estro em cabras da raça Toggenburg com dois diferentes dispositivos intravaginais. Arq. Bras. Med. Vet. Zoot., v.58, p.367-372, 2006.

MENCHACA, A.; RUBIANES, E. Relation between progesterone concentrations during the luteal phase and follicular dynamics in goats. Theriogenology, v.57, p.1411-1419, 2002.

NAGY, P.; GUILLAUME, D.; DAELS, P. Seasonality in mares. Anim. Reprod. Sci., v.6061, p.245-262, 2000.

NASCIMENTO, P.M.P. Indução de estro sincronizado em cabras da raça Toggenburg com protocolos de curta, média e longa durante $o$ anestro estacional e acasalamento natural. 2009. 85f. Dissertação (Mestrado em Medicina Veterinária) - Universidade Federal Fluminense, Niterói, RJ.
ORIHUELA, A. Some factors affecting the behavioral manifestation of oestrus in cattle: a review. Apll. Anim. Behav. Sci., v.70, p.1-16, 2000.

PIETROSKI, A.C.C.A.; BRANDÃO, F.Z.; SOUZA, J.M.G. et al. Short, medium and longterm hormonal treatments for induction if synchronized strus and ovulation in Saanen goats during the nonbreeding season. Rev. Bras. Zootec., v.42, p.168-173, 2013.

RUBIANES, E.; CASTRO, T.; KMAID, S. Estrus response after a short progesterone priming inseasonally anestrous goats. Theriogenology, v.49, p.356, 1998.

SOUZA, J.M.G.; TORRES, C.A.; MAIA, A.L.R.S. et al. Autoclaved, previously used intravaginal progesterone devices induces estrus and ovulation in anestrous Toggenburg goats. Anim. Reprod. Sci., v.129, p.50-55, 2011.

SOUZA, J.M.G.; TORRES, C.A.A.; MAIA, A.L.R.S. et al. Autoclaved, previously used intravaginal progesterone devices induces estrus and ovulation in anestrous Toggenburg goats. Anim. Reprod. Sci., v.129, p.50-55, 2013.

SUITER, J. Body condition score of sheep and goat. Farm Note, n.69, 1994.

TENÓRIO FILHO, F.; SANTOS, M.H.B.; CARRAZZONI, P.G. et al. Follicular dynamics in Anglo-Nubian goats using transrectal and transvaginal ultrasound. Small Ruminant Res., v.72, p.51-56, 2007.

TRALDI, A.S.; LOUREIRO, M.F.P.; CAPEZZUTO, A. et al. Métodos de controle da atividade reprodutiva em caprinos. Rev. Bras. Reprod. Anim., v.31, p.254-260, 2007.

ZAMBRINI, F.N. Dinâmica ovulatória $e$ inseminação artificial em tempo prédeterminação em cabras com estro induzido. 2006. 44f. Dissertação (Mestrado em Medicina Veterinária) - Universidade Federal de Viçosa, Viçosa, MG. 